\title{
Lower Bounds for the Cop Number when the Robber is Fast
}

\author{
Abbas Mehrabian \\ Department of Combinatorics and Optimization \\ University of Waterloo \\ amehrabian@uwaterloo.ca
}

\begin{abstract}
We consider a variant of the Cops and Robbers game where the robber can move $t$ edges at a time, and show that in this variant, the cop number of a $d$-regular graph with girth larger than $2 t+2$ is $\Omega\left(d^{t}\right)$. By the known upper bounds on the order of cages, this implies that the cop number of a connected $n$-vertex graph can be as large as $\Omega\left(n^{2 / 3}\right)$ if $t \geq 2$, and $\Omega\left(n^{4 / 5}\right)$ if $t \geq 4$. This improves the $\Omega\left(n^{\frac{t-3}{t-2}}\right)$ lower bound of Frieze, Krivelevich, and Loh (Variations on Cops and Robbers, preprint, 2010) when $2 \leq t \leq 6$. We also conjecture a general upper bound $O\left(n^{t / t+1}\right)$ for the cop number in this variant, generalizing Meyniel's conjecture.
\end{abstract}

\section{Introduction}

The game of Cops and Robbers, introduced by Nowakowski and Winkler [10] and independently by Quilliot [11, is a perfect information game played on a finite graph $G$. There are two players, a set of cops and a robber. Initially, the cops are placed onto vertices of their choice in $G$ (where more than one cop can be placed at a vertex). Then the robber, being fully aware of the cops placement, positions herself in one of the vertices of $G$. Then the cops and the robber move in alternate rounds, with the cops moving first; however, players are permitted to remain stationary on their turn if they wish. The players use the edges of $G$ to move from vertex to vertex. The cops win and the game ends if eventually a cop steps into the vertex currently occupied by the robber; otherwise, i.e., if the robber can elude the cops indefinitely, the robber wins. The parameter of interest is the cop number of $G$, which is defined as the minimum number of cops needed to ensure that the cops can win. We will assume that the graph $G$ is simple and connected, because deleting multiple edges or loops does not affect the set of possible moves of the players, and the cop number of a disconnected graph obviously equals the sum of the cop numbers for each connected component.

For a survey of results on the cop number and related search parameters, see the survey by Hahn 7 . The most well known open question in this area is Meyniel's conjecture, published by Frankl in [5]. It states that for every graph $G$ on $n$ vertices, $O(\sqrt{n})$ cops are enough to win. This is asymptotically tight, i.e. for every $n$ there exists an $n$-vertex graph with cop number $\Omega(\sqrt{n})$. The best upper bound found so far is $n 2^{-(1-o(1)) \sqrt{\log _{2} n}}$ (see [6, 9, 12] for several proofs). 
Here we consider the variant where in each move, the robber can take any path of length at most $t$ from her current position, but she is not allowed to pass through a vertex occupied by a cop. The parameter $t$ is called the speed of the robber. This variant was first considered by Fomin, Golovach, Kratochvíl, Nisse, and Suchan [4, who proved that computing the cop number is NP-hard for every $t$. Next, Frieze, Krivelevich, and Loh [6] showed that the cop number of an $n$-vertex graph can be as large as $\Omega\left(n^{\frac{t-3}{t-2}}\right)$. They also asked whether there exist graphs with cop number $\omega(\sqrt{n})$ if $t=2$. We give a positive answer to this question, proving the existence of graphs with cop number $\Omega\left(n^{2 / 3}\right)$ for $t \geq 2$, and graphs with cop number $\Omega\left(n^{4 / 5}\right)$ for $t \geq 4$. This improves their bound $\Omega\left(n^{\frac{t-3}{t-2}}\right)$ when $2 \leq t \leq 6$. In Section 2 the lower bounds are proved, and in Section 3 a conjecture is proposed, predicting the asymptotic value of cop number in this general setting.

\section{The lower bounds}

Lemma 1. Let $t, d$ be positive integers with $t \leq d+1, G$ be $a(d+1)$-regular graph with girth larger than $2 t+2$ and $\alpha \in(0,1)$ be such that $\alpha d^{t}$ is an integer. Assume that the robber has speed $t$. Then the cop number of $G$ is at least $\frac{\alpha(1-\alpha) d^{2 t}}{2(t+2)(d+1)^{t}}$.

Proof. Let us first define a few terms. A cop controls a vertex $v$ if the cop is on $v$ or on an adjacent vertex. A cop controls a path if it controls a vertex of the path. The cops control a path if there is a cop controlling it. A vertex $r$ is safe if there exists a set $S$ of vertices of size $\alpha d^{t}$ such that for each $s \in S$, there is an $(r, s)$-path of length $t$ not controlled by the cops.

Assume that there are less than $\frac{\alpha(1-\alpha) d^{2 t}}{2(t+2)(d+1)^{t}}$ cops in the game, and we will show that the robber can elude forever. We may assume that the cops all start in one vertex $u$, and the robber starts in a vertex $v$ at distance $t+1$ from $u$. Let $N$ be the set of vertices at distance $t$ from $v$. Then by the girth condition, the cops control only one vertex from $N$, and since $|N|>d^{t}, v$ is a safe vertex. Hence we just need to show that if the robber is in a safe vertex before the cops move, then she can move to a safe vertex after the cops move.

Assume that the robber is in a safe vertex $r$ after her last move. Then by definition there exists a set $S$ of vertices of size $\alpha d^{t}$ such that for each $s \in S$, there is an $(r, s)$-path of length $t$ not controlled by the cops. Let $U$ be the set of all vertices of these paths. Now, look at the situation after the cops move. There is no cop in $U$, thus the robber can move to any of the vertices in $S$ in her turn, and it suffices to prove that there is a safe vertex in $S$. Note that the girth of the graph is larger than $2 t+2$, so $S$ is an independent set and no vertex outside $U$ is adjacent to two distinct vertices of $S$. By an escaping path we mean a path of length $t$ with its first vertex in $S$ and second vertex not in $U$. Clearly every $s \in S$ is the starting vertex of exactly $d^{t}$ escaping paths.

Claim. After the cops move, each cop controls at most $(t+2)(d+1)^{t}$ escaping paths.

Proof. We first prove that every vertex $v$ is on at most $t(d+1)^{t-1}+(d+1)^{t}$ escaping paths, and if $v \notin S$ then $v$ is on at most $t(d+1)^{t-1}$ escaping paths. Let $u_{1} u_{2} u_{3} \ldots u_{t+1}$ be an escaping path with $u_{1} \in S$ and $u_{2} \notin U$ such that $v$ is its $i$-th vertex, i.e. $v=u_{i}$. Assume first that $i \neq 1$. Note that by definition we have $u_{2} \notin U$, so $u_{1}$ is determined uniquely by $u_{2}$. There are (at most) $d+1$ choices for each of $u_{i-1}, \ldots, u_{2}$, 
and for each of $u_{i+1}, u_{i+2}, \ldots, u_{t+1}$. Consequently, for each $2 \leq i \leq t+1, v$ is the $i$-th vertex of at most $(d+1)^{t-1}$ escaping paths, so if $v \notin S$ then $v$ is on at most $t(d+1)^{t-1}$ escaping paths. If $i=1$ then $v \in S$ and there are at most $d+1$ choices for each of $u_{2}, u_{3}, \ldots, u_{t+1}$, thus each $v \in S$ is the first vertex of at most $(d+1)^{t}$ escaping paths. This shows that $v$ is on at most $t(d+1)^{t-1}+(d+1)^{t}$ escaping paths.

Since the robber was in a safe vertex before the cops move, no cop is in $U$ at this moment. Hence, each cop can control at most one vertex from $S$, through which he can control at most $(d+1)^{t}+t(d+1)^{t-1}$ escaping paths. Through every other vertex he can control at most $t(d+1)^{t-1}$ escaping paths, and he controls $d+2$ vertices in total. Therefore he controls no more than $(d+1)^{t}+(d+2) t(d+1)^{t-1} \leq(t+2)(d+1)^{t}$ escaping paths.

Now, since there are less than $\frac{\alpha(1-\alpha) d^{2 t}}{2(t+2)(d+1)^{t}}$ cops in the game, the cops control less than $\alpha(1-\alpha) d^{2 t} / 2$ of the escaping paths. Since $S$ has $\alpha d^{t}$ vertices, and each path has two endpoints, there must be an $s \in S$ such that at most $(1-\alpha) d^{t}$ escaping paths starting from $s$ are controlled. Consequently, there are $\alpha d^{t}$ uncontrolled escaping paths starting from $s$. Note that girth of $G$ is larger than $2 t$ so the other endpoints of these paths are distinct. Hence $s$ is safe by definition and the robber moves to $s$.

Corollary 1. Let $t$ be some fixed positive integer denoting the speed of the robber. If $G$ is a d-regular graph (where $d \geq \max \{3, t\}$ ) with girth larger than $2 t+2$, then the cop number of $G$ is $\Omega\left(d^{t}\right)$.

In order to use Corollary 1 to prove interesting lower bounds for the cop number, one should look at vertex-minimal regular graphs with large girth, known as cages. Here are two useful results on cages (see [3] for a survey):

Theorem 1 ([8]). Let $g \geq 5$, and $d \geq 3$ be an odd prime power. Then there exists a d-regular graph of girth $g$ with at most $2 d^{1+\frac{3}{4} g-a}$ vertices, where $a=4,11 / 4,7 / 2,13 / 4$ for $g \equiv 0,1,2,3(\bmod 4)$, respectively.

Theorem 2 ([2]). Let $d \geq 3$ be a prime power. Then there exists a d-regular graph with girth 12 and at most $2 d^{5}$ vertices.

Theorem 3. Let $t$ be some fixed positive integer denoting the speed of the robber.

(a) If $t \geq 2$ then for every $n$ there exists an $n$-vertex graph with cop number $\Omega\left(n^{2 / 3}\right)$.

(b) If $t \geq 4$ then for every $n$ there exists an n-vertex graph with cop number $\Omega\left(n^{4 / 5}\right)$.

Proof. (a) As the cop number will not decrease when the speed of the robber is increased, we just need to show the proposition for $t=2$. Let $n \geq 54$ and $d$ be the largest prime number such that $2 d^{3} \leq n$. Since there exists a prime between $d$ and $2 d$, we have $n<2(2 d)^{3}$ so $d=\Theta\left(n^{1 / 3}\right)$. By Theorem 11, there exists a $d$-regular graph $H$ of girth 7 with at most $2 d^{3}$ vertices. By Corollary 1 the cop number of $H$ is $\Omega\left(d^{2}\right)=\Omega\left(n^{2 / 3}\right)$. Let $G$ be the graph formed by joining some vertex of $H$ to an endpoint of a disjoint path with $n-|V(H)|$ vertices. It is easy to check that the cop number of $G$ equals the cop number of $H$, which is $\Omega\left(n^{2 / 3}\right)$.

(b) Again we just need to show the proposition for $t=4$. Let $n \geq 486$ and $d$ be the largest prime number such that $2 d^{5} \leq n$. A similar argument shows that $d=\Theta\left(n^{1 / 5}\right)$. By Theorem 2, there exists a $d$-regular graph $H$ of girth 12 with at most $2 d^{5}$ vertices. By Corollary 1 the cop number of $H$ is $\Omega\left(d^{4}\right)=\Omega\left(n^{4 / 5}\right)$. Let $G$ be the graph formed by joining some vertex of $H$ to an endpoint of a 
disjoint path with $n-|V(H)|$ vertices. Then the cop number of $G$ equals the cop number of $H$, which is $\Omega\left(n^{4 / 5}\right)$.

\section{Concluding remarks}

Let $f_{t}(n)$ be the maximum possible cop number of a connected $n$-vertex graph assuming the robber has speed $t$. It is well-known (and also follows from Corollary 1 and Theorem 1 with $g=5$ ) that $f_{1}(n)=\Omega(\sqrt{n})$. Meyniel conjectured that indeed $f_{1}(n)=\Theta(\sqrt{n})$. Frieze, Krivelevich, and Loh [6] showed that $f_{t}(n)=\Omega\left(n^{\frac{t-3}{-2}}\right)$ if $t \geq 3$. In this note we proved that $f_{2}(n)=\Omega\left(n^{2 / 3}\right)$ and $f_{4}(n)=\Omega\left(n^{4 / 5}\right)$. A natural question is that of the asymptotic behavior of $f_{t}(n)$.

Notice that if $G$ is a $d$-regular graph with girth larger than $2 t+2$, then Moore's bound gives $d=$ $O\left(n^{1 / t+1}\right)$. Hence Corollary 1 cannot give a better bound than $f_{t}(n)=\Omega\left(n^{t / t+1}\right)$. Generalizing Meyniel's conjecture, we conjecture that this is actually the asymptotic behavior of $f_{t}(n)$.

Conjecture. For every fixed $t$ we have $f_{t}(n)=\Theta\left(n^{t / t+1}\right)$.

Proving better upper bounds on the order of cages would imply that the conjecture is tight. Specifically, if for a fixed $t$, and infinitely many $d$, there exists a $d$-regular graph with girth larger than $2 t+2$ on $O\left(d^{t+1}\right)$ vertices, then $f_{t}(n)=\Omega\left(n^{t / t+1}\right)$ (see Corollary 1).

Acknowledgement. The author thanks Nick Wormald for his suggestions on improving the presentation.

Addendum. Alon and the author [1] have recently extended the result of this note, and proved that $f_{t}(n)=\Omega\left(n^{t / t+1}\right)$ for every fixed positive integer $t$.

\section{References}

[1] N. Alon and A. Mehrabian, On a generalization of Meyniel's conjecture on the Cops and Robbers game, Electron. J. Combin. 18 (2011), no. 1, Research Paper 19, 7 pp. (electronic).

[2] G. Araujo, D. González, J. J. Montellano-Ballesteros, and O. Serra, On upper bounds and connectivity of cages, Australas. J. Combin. 38 (2007), 221-228. MR 2324289 (2008c:05086)

[3] G. Exoo and R. Jajcay, Dynamic cage survey, Electron. J. Combin. 15 (2008), Dynamic Survey 16, 48 pp. (electronic).

[4] F. V. Fomin, P. A. Golovach, J. Kratochvíl, N. Nisse, and K. Suchan, Pursuing a fast robber on a graph, Theoret. Comput. Sci. 411 (2010), no. 7-9, 1167-1181. MR 2606052

[5] P. Frankl, Cops and robbers in graphs with large girth and Cayley graphs, Discrete Appl. Math. 17 (1987), no. 3, 301-305. MR 890640 (88f:90204)

[6] A. Frieze, M. Krivelevich, and P. Loh, Variations on cops and robbers, arXiv:1004.2482v1 [math.CO]. 
[7] G. Hahn, Cops, robbers and graphs, Tatra Mt. Math. Publ. 36 (2007), 163-176. MR 2378748 (2009b:05254)

[8] F. Lazebnik, V. A. Ustimenko, and A. J. Woldar, New upper bounds on the order of cages, Electron. J. Combin. 4 (1997), no. 2, Research Paper 13, approx. 11 pp. (electronic), The Wilf Festschrift (Philadelphia, PA, 1996). MR 1444160 (98e:05066)

[9] L. Lu and X. Peng, On Meyniel's conjecture of the cop number, submitted, 2009.

[10] R. Nowakowski and P. Winkler, Vertex-to-vertex pursuit in a graph, Discrete Math. 43 (1983), no. 2-3, 235-239. MR 685631 (84d:05138)

[11] A. Quilliot, Jeux et pointes fixes sur les graphes, Ph.D. thesis, Université de Paris VI, 1978.

[12] A. Scott and B. Sudakov, A new bound for the cops and robbers problem, arXiv:1004.2010v1 [math.CO]. 